\title{
An experimental and numerical study of the particle dispersion in a ventilated room
}

\author{
M. Gustiuc ${ }^{1}$, T. Denes ${ }^{1}$, S. El Hamdani ${ }^{1}$, K. Limam ${ }^{1}$ \& I. Colda ${ }^{2}$ \\ ${ }^{1}$ LEPTAB, University of La Rochelle, France \\ ${ }^{2}$ Technical University of Constructions, Bucharest
}

\begin{abstract}
This paper focuses on the solid contaminant dispersion in a ventilated room under different ventilation conditions. Experiments in a full-scale laboratory chamber are conducted in order to validate the numerical results. The ventilation efficiency for different positions of the inlet and outlet, as well as the influence of the ventilation rate, was established. A two-layer k- $\varepsilon$ high Reynolds number is used for the continuous phase, with the same boundaries and initial conditions the experimental setup. The 113000 particles in the range of $0.35-10 \mu \mathrm{m}$ are tracked during the $600 \mathrm{~s}$ simulation time. The importance of the gravity forces for such small particles is established.
\end{abstract}

Keywords: CFD modelling, airflow rate, building, particle, Eulerian, Lagrangian, ventilation, experimental, numerical.

\section{Introduction}

The indoor air quality became a major concern in the last years. People spend most of their time in closed spaces, therefore is vital to predict the pollutant concentration as accurate as possible.

Researches were conducted under various conditions, but the complexity and the variety of the phenomena involved in the pollutant dispersion in indoor spaces makes difficult to achieve reliable results. Many of the earlier studies considered well-mixed conditions for the contaminant, as Nazaroff et al. [1], Nomura et al. [2], Lai et al. [3], Abadie et al. [4]. This approach means that the concentration decay rate in one point fully describe the entire room. Adequacy of the well mixed hypothesis depends on a variety of factors, such as integration time and environmental determinants. Room's ventilation, temperature 
distribution and furniture configuration are key factors, Richmond-Bryant et al. [5], Elisabeth et al. [6], Schneider et al. [7] and Thatcher et al. [8]. These researches reported that certain conditions could lead to preferential concentrations in interior spaces or to non-uniform contaminant dispersion. In addition, the aerodynamic radius will increase by the value of relative humidity. Pyykönen and Jokiniemi [9] observed that particle's diameter can grow from $1 \mu \mathrm{m}$ to $4 \mu \mathrm{m}$ during condensation. We consider for the indoor airflow that the humidity effects could be neglected if its value does not exceed $70 \% \mathrm{HR}$, as mentioned by Busch et al. [12] and Einberg and Holmberg [13]. Particles respond more or less to the carrier fluid fluctuations as there relative density and shape plays a major role. For instance, Zhao and Guan [14] considered that, in the case of $1000 \mathrm{~kg} / \mathrm{m}^{3}$ density particle in a personalized ventilated room, passive contaminants could be considered those that aerodynamically diameter would not exceed $2 \mu \mathrm{m}$.

Another important parameter is the particle loading. The continuous and disperse phase are interdependent. If one-way coupling is less computational expensive, for high loadings we could not neglect the influence of the dispersed phase on the continuous one, Shirolkar et al. [10], Gouesbet and Berlement [11]. To introduce the two-way coupling in RANS simulations of the fluid, the turbulence models are modified by adding some source terms in the balance equations for momentum, turbulence energy and dissipation. If the numberdensity of particles is big enough, the particles will interact in an indirect way through hydrodynamic forces or directly, by collisions or coalescence.

Our study, both numerical and experimental, focuses on the contaminant removal efficiency in a full-scale laboratory wooden-made chamber under isothermal conditions. Alumina powder with the D50 aerodynamically diameter of $0.65 \mu \mathrm{m}$ and $0.85 \mu \mathrm{m}$ was injected and homogenised before the measurement starts. A buffer zone assures constant temperature conditions. A two-layer k- $\varepsilon$ high Reynolds numbers and a Lagrangian particle tracking technique account for respectively continuous and dispersed phase. Only one-way coupling is considered, as a common assumption in building environment simulation strategy. The last part investigates the importance of the gravitational forces function of the particle size.

\section{Experimental setup}

Experiments are conducted in a $9.38 \mathrm{~m}^{3}$ wooden-made test-cell, fig. 1 . A buffer zone assures a relatively stable interior temperature and humidity, as 8 PT100 temperature sensors and 3 temperature/hygrometric sensors continuously monitories this parameters. The PT100 sensors are placed into the wall's vicinity and for data acquisition we used AOiP SA32 device. There are minimal changes in the temperature and the humidity during the measurements. The $7 \times 49 \mathrm{~cm}^{2}$ inlet and outlet are opposite wall mounted. The facility is carefully sealed up, as SF6 tests prove low infiltration values. Two methods were used for the air exchange rate, the tracer gas and the direct velocity measurements in the inflow pipe. The mean air inlet velocity was taken as the mean value for the two cases. 
Three laser particle counters, which can each measure aerodynamically diameters between $0.3 \mu \mathrm{m}-20 \mu \mathrm{m}$, are equidistantly placed in the median plane as shown in the fig. 1. Two Alumina powder of $0.65 \mu \mathrm{m}$ and $0.85 \mu \mathrm{m}$ as well as orgasol powder of $10 \mu \mathrm{m}$ centred were injected with a TOPAS dust injector. Before the injection, particles were electrical neutralised with a TOPAS EAN 581 electrical neutraliser.

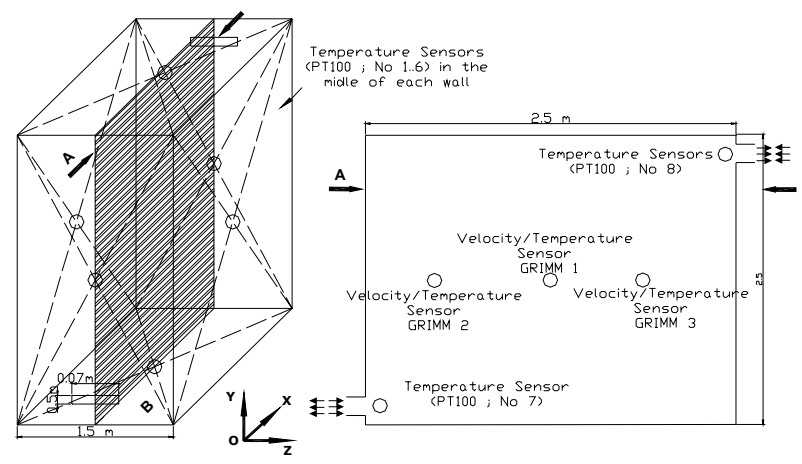

Figure 1: Experimental facility.

The influence of both airflow rates of $1 \mathrm{ACH}$ and $3.7 \mathrm{ACH}$, and the inlet and the outlet location were studied by mean concentration first order decay rate, Nazaroff et al. [1]. For each particle size interval, we obtain by tracing the exponential fit curve, the particle loss rate coefficient:

$$
C(t)=C_{0} \exp (-\beta t) .
$$

This equation stands for the zero particle concentration at the inlet and no interior sources after particle injection. The particle concentration was high enough to accurately measure the concentration decay, but not so high that coagulation would be appreciable.

The experimental protocol is as follows:

- the optical counters are positioned at their locations and a interior ceiling fan is turned on and the inlet and outlet are sealed to;

- particles are injected in the test chamber trough the electrical neutralization device;

- after approximately 12 seconds the particle injection and the fan are stopped, the inlet and the outlet are sealed off and the ventilation turned on.

The global deposition coefficient $\beta$ contains any mechanism responsible for the indoor concentration decay. If we consider homogenous contaminant concentration, the difference between $\beta$ and the ventilation rate gives the wall deposition coefficient $\beta_{d}$. As explained by Bouilly [16], we can measure the 
deposition phenomenon by calculating the mean deposition velocity, $v_{d}=\frac{V}{S}$, where $V$ is the chamber's volume and $S$ is the total deposition surface.

\section{Computational model}

The computational models for predicting the contaminant particle distribution in a building need two numerical parts. Firstly, an airflow pattern must been known before calculating the distribution of the contaminant particle in the building. Then, substituting the already known velocity fields into the equation of particle motion, a complete particle trajectory can be obtained. As only the one-way coupling is considered, we first achieve the steady state conditions for the airfield without particle loading and then we inject the particles. Therefore, we obtained a substantial reduction in computational time. Our model is based on a commercial CFD code, STAR-CD, which is a finite-volume Navier-Stokes solver with multiphase capabilities.

\subsection{Primary flow}

The main CFD airflow modelling principles are as follows: computational domain discretisation, turbulence modelling, treatment of the airflow near the wall and boundary conditions. The standard k- $\varepsilon$ turbulence model is employed, as it was tested with good results in many other cases. The Reynolds-averaged conservation equation along with the equations for the turbulent kinetic energy and the dissipation rate can be written in a general form below, with the coefficients given in the table 1 :

$$
\frac{\partial}{\partial t}\left(A_{i} \Phi\right)+\frac{\partial}{\partial x_{j}}\left(B_{i} \Phi\right)-\frac{\partial}{\partial x_{j}}\left(\Gamma \frac{\partial \Phi}{\partial x_{j}}\right)=S
$$

Table 1: $\quad$ General equations.

\begin{tabular}{|c|c|c|c|c|c|}
\hline & $\Phi$ & $A_{i}$ & $B_{i}$ & $\Gamma_{i}$ & $S$ \\
\hline Continuity equation & 1 & 0 & $\rho^{g} u_{j}^{g}$ & 0 & 0 \\
\hline Momentum equation & $u_{i}^{g}$ & 0 & $\rho^{g} u_{j}^{g}$ & $\mu_{t}^{g}$ & $-\frac{\partial P}{\partial x_{j}}$ \\
\hline $\begin{array}{c}\text { Turbulent kinetic } \\
\text { energy equation }\end{array}$ & $k^{g}$ & 0 & $\rho^{g} u_{j}^{g}$ & $\frac{\mu_{t}^{g}}{\sigma_{k}}$ & $P_{k}-\rho^{g} \varepsilon^{g}$ \\
\hline Dissipation equation & $\varepsilon^{g}$ & 0 & $\rho^{g} u_{j}^{g}$ & $\frac{\mu_{t}^{g}}{\sigma_{\varepsilon}}$ & $\frac{\varepsilon^{g}}{k^{g}}\left(C_{\varepsilon 1} P_{k}-C_{\varepsilon 2} \rho^{g} \varepsilon^{g}\right)$ \\
\hline
\end{tabular}


The source term from the turbulent kinetic energy equation represents the turbulent generation by shear and normal stresses and the viscous dissipation; $\sigma_{k}, \sigma_{\varepsilon}$ are the turbulent Prandtl number. The default values are given in the table 2 .

Table 2: $\quad$ Coefficients of the standard k- $\varepsilon$ model.

\begin{tabular}{|l|l|l|l|}
\hline$\sigma_{k}$ & $\sigma_{\varepsilon}$ & $C_{\varepsilon 1}$ & $C_{\varepsilon 1}$ \\
\hline 1 & 1.22 & 1.44 & 1.92 \\
\hline
\end{tabular}

The model uses a PISO algorithm coupling with a monotone advection and reconstruction scheme (MARS) and first-order up-wind schemes respectively for velocity an scalar terms.

The high Reynolds number turbulence models are not valid near the wall, where molecular and turbulence effects are of comparable magnitude. The use of the wall functions needs a priori estimation of the boundary layer width, which is variable along the solid boundary. To overpass this problem, we used a two-layer model with non-slip velocity conditions. A one-equation $\mathrm{k}-l$ turbulence model developed by Norris and Reynolds [15], as given in table 3, is applied within some cells of the near-wall-layer; a switching function translates to the interior domain equations.

Table 3: $\quad$ Two-layer turbulence model.

\begin{tabular}{|l|l|l|}
\hline $\begin{array}{c}\text { Turbulent dissipation } \\
\text { Rate function }\end{array}$ & $f_{\mu}$ function & Constants \\
\hline$\varepsilon=\frac{k^{3 / 2}}{l}\left(1+\frac{C_{\varepsilon}}{\operatorname{Re}_{y}}\right)$ & $f_{\mu}=1-\exp \left(-\frac{1}{A_{\mu}} \operatorname{Re}_{y}\right)$ & $\begin{array}{l}A_{\mu}=50.5 \\
C_{\varepsilon}=2.3 \\
\end{array}$ \\
& $\begin{array}{l}\operatorname{Re}_{y}=\frac{\sqrt{k} y}{v} \\
\\
\end{array}$ & \\
\hline$=\kappa C_{\mu}^{-0.75} y$ & \\
\hline
\end{tabular}

As we have an isothermal flow, the centre of the first cell near wall is located between 4 and 8 wall functions. This was considered a good compromise between the computational cost and the solution accuracy. The solution was computed only with on half of the geometrical domain, as we introduced the symmetry plan showed in the fig. 1. Grid independency tests gives us an over all grid-independent results within 219000 cells.

\subsection{Dispersed phase}

To resolve the dispersed phase we used a point-volume Lagrangian tracking technique, which is the natural frame of treating particles. The particle trajectory 
can be determined by solving its equation of motion. This equation can be obtained from Newton's second Law of Motion:

$$
m_{d} \frac{d u_{d}}{d t}=F_{d r}+F_{p}+F_{a m}+F_{b}
$$

where, $F_{d r}=\frac{1}{2} C_{d} \varphi^{g} A_{d}\left|u-u_{d}\right|\left(u-u_{d}\right)$ is the drag force, $F_{P}=-V \nabla p$ is the pressure force, $F_{a m}=-C_{a m} \varphi V_{d} \frac{d\left(u_{d}-u\right)}{d t}$ is the virtual mass force and $F_{b}=m_{d}\left[g-\omega \times(\omega \times r)-2\left(\omega \times u_{d}\right)\right]$ is a general body force.

Knowledge of the droplet velocity allows its instantaneous position vector to be determined. A stochastic velocity generation deals with the turbulent particle dispersion. It is assumed that the fluctuating velocity within each eddy is isotropic and obeys a Gaussian probability density function. The eddy interaction time $\tau_{I}$ assumed to be sufficient short that the fluid velocity in a given eddy constant. The interaction time is taken to be the smaller of the eddy lifetime $\tau_{e}$ and the time required for the droplet to traverse the eddy $\tau_{t}$.

The momentum equation, eqn. (2), is discretised into first-order Euler-implicit form, with the time step $\delta t_{d}$ determined in the following manner:

$$
\delta t_{d}=\min \left(\delta t, \delta t_{c}, \tau_{M}, \tau_{m}, \tau_{t}\right)
$$

where $\delta t$ is the carrier phase time step, $\delta t_{c}$ is the cell droplet time scale, $\tau_{M}$, $\tau_{m}$, are the momentum and mass relaxation time scales.

The particle injection happens only after the flow field reaches steady conditions. Therefore, the user defined time step do not influence the calculations, being used only fore the data post treatment.

\subsection{Boundary and initial conditions}

The boundary conditions for the inlet are specified empirically. The turbulence intensity and characteristic length are estimated in order to obtain the turbulent energy and the dissipation rate at the inlet and a constant velocity field is taken into account for all the cases. All the influx is exhaust by the outlet. No-slip boundary conditions stand for particle-structure interaction.

A large number of 113000 solid spherical particles are uniformly injected in the flow with zero initial velocity. As we do not consider two-way or four-way coupling, concentration fields are obtained by, separately for each particle diameter of $0.35 \mu \mathrm{m}, 1.5 \mu \mathrm{m}, 2.5 \mu \mathrm{m}$ and $10 \mu \mathrm{m}$. The particle-wall interaction is perfect elastic rebound. 


\section{Results and discussion}

A common way to estimate the ventilation efficiency is by the means of the first order concentration decay coefficient. One point concentration measurement allows us to evaluate different ventilation strategies and the mean wall deposition velocity. This method generally stands for the perfect mixing case. Other two particle counters are used to validate the pollutant homogeneity; relative variations are found les than $6 \%$ for different points in the test chamber for the low ventilation rate case. We can see in fig. 2 , in the case of low ventilation rate, that the decay coefficient and the mean deposition velocity increase with the aero dynamical particle diameter. Other researches suggest the same behaviour, Lai [17]. An unexpected trend is observed for the two inlet/outlet cases. The concentration decay rate and consequently the deposition velocity for the bigger particles seem to be more important in the case of the inlet situated in the lower region. The numerical results, fig. 3, suggest that, while the button-top ventilation strategy oppose the gravitational settling and enhance the pollutant mixing resulting in a better particle removal, the top-bottom strategy accelerates the settling behaviour but somehow keep the particle away from the exhaust. This results in poor removal efficiency for the bigger particles in the top-bottom ventilation case.

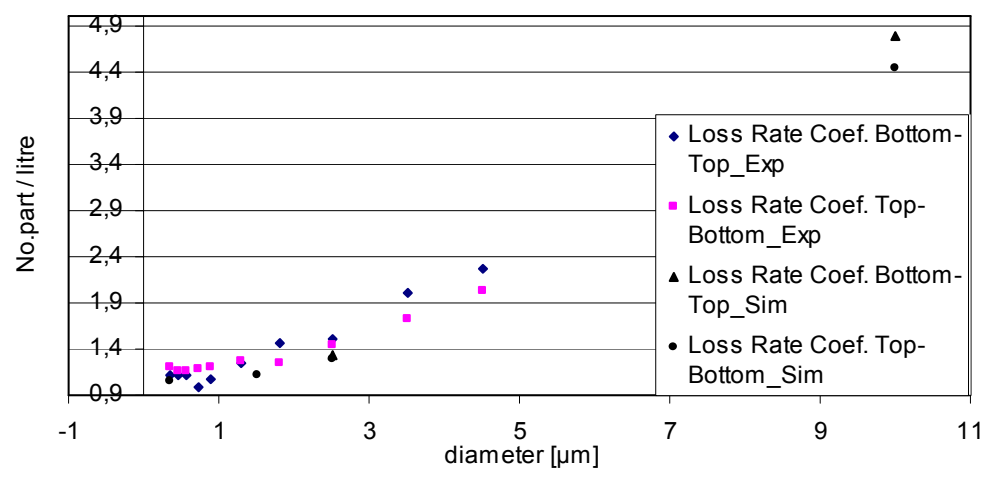

Figure 2: $\quad$ Global loss rate coefficient for low ventilation rate case $\left(1 \mathrm{~h}^{-1}\right)$.

The perfect mixing assumption is no longer valid in the case of 4 air change ventilation rate. Experimental measurement show concentration differences up to $20 \%$ in the test facility. The concentration decay coefficients for the 3 particle counters proves different decay behaviour in different zones of the room. The simulations results, fig. 4 median plane, show a strong particle concentration in the middle of the room. The darkest regions for the turbulent kinetic energy are those of the smallest magnitude. The pollutant accumulation area seems to superpose on a low mean velocity and kinetic turbulent energy zone. Our numerical model seems to overestimate this trend, as concentrations differences are up to $28 \%$ grater than the experimental measurements for the measurement 
locations. One possible reason is that the $\mathrm{k}-\varepsilon$ model over predicts turbulence kinetic energy which can lead to a grater particle dispersion.

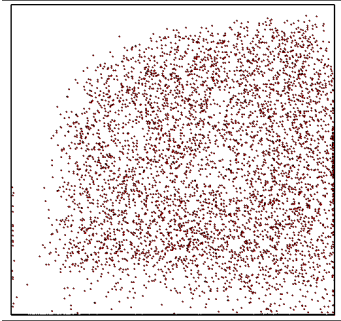

a) Bottom-top ventilation.

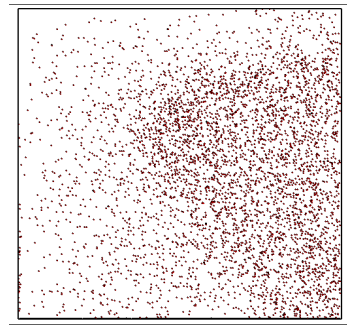

b) Top-bottom ventilation.

Figure 3: Particle distribution in a longitudinal plane for two low rate ventilation strategies.

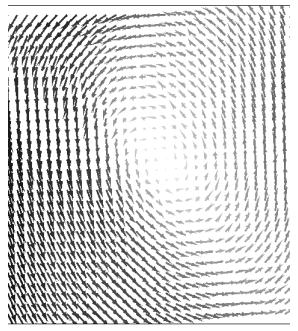

a) Velocity.

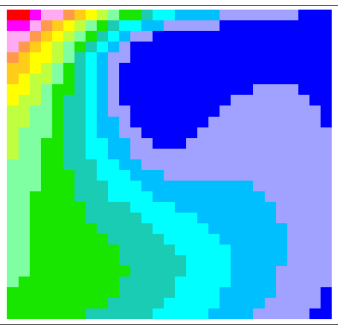

b) Turbulent kinetic energy.

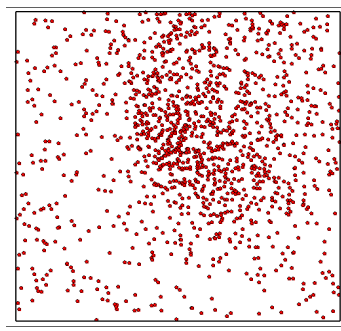

c) Particles distribution.

Figure 4: $\quad$ High ventilation rate $\left(3.7 \mathrm{~h}^{-1}\right), 0.35 \mu \mathrm{m}$ particle diameter.

We also tested the effect of the buoyancy force on the particle concentration. The pollutant accumulation process is less important in the absence of the gravity force, but the trend rests the same.

\section{Conclusion}

This study demonstrates the crucial importance of the CFD pollutant prediction. We have seen that the increase of the ventilation rate may lead to preferential concentration and we can no longer consider perfect pollutant mixing. The mono zone approach in concentration prediction is a cheap model that can give good results, but the complexity of the fluid/particle physics could make it a dangerous technique. 


\section{References}

[1] Nazaroff, W.W, Gadgil, A.J. \& Wescheler, C.J., Critique of the Use of Deposition Velocity in Modelling Indoor Air Quality, Modeling Indoor Air Quality and Exposure, ASTM STP 1205, pp. 81-104, 1993.

[2] Nomura, Y., Hopke, P.K., Fitzgerald, B., \& Mesbah, B., Deposition of Particles in a Chamber as a Function of Ventilation Rate, Aerosol Science and Technology, 27, pp. 62-72, 1997.

[3] Alvin Lai C.K., Byrne Miriam, A. \& Godard Antony J.H., Experimental Studies of the Effect of Rough Surfaces and Air Speed on Aerosol Deposition in a test Chamber, Aerosol Science and Technology, 36, pp. 973-982, 2002.

[4] Abadie M., Limam K. \& Francis A., Indoor particle pollution: effect of wall textures on particle deposition, Building and Environment, 36, pp. 821-827, 2001.

[5] Richmond-Bryant, J., Eisner, A.D., Brixey, L.A. \& Wiener, R.W., Shortterm dispersion in of indoor aerosols: cant it be assumed the room is well mixed?. Building and Environment, 41, pp. 156-163, 2004.

[6] Elisabeth, M., Non-buoyant pollutant sources and particles in displacement ventilation, Building and environment, 36, pp. 829-836, 2001.

[7] Schneider, T., Kildesø, J. \& Berum, N.O., A two compartment model for determining the contribution of sources, surface deposition and resuspension to air and surface dust concentration levels in occupied rooms, Building and Environment, 34, pp. 583-595, 1999.

[8] Thatcher, L.T., Lai, A.C.K., Moreno-Jackson, R., Sextro, R.G. \& Nazaroff, W.W., Effects of room furnishing and air speed on particle deposition rates indoors, Atmospheric and Environment, 36, pp. 18111819, 2002.

[9] Pyykönen, J., \& Jokiniemi, J., Computational Fluid Dynamics based sectional aerosol modelling schemes, Journal of Aerosol Science, 31, pp. 531-550, 2000.

[10] Shirolkar, J.S., Coimbra, C.F.M. \& Queiroz McQuay, M., Fundamental Aspects of Modeling Turbulent Particle Dispersion in Dilute Flows, Progress in Energy and Combustion Science, 22, pp. 363-399, 1996.

[11] Gouesbet, G. \& Berlement, A., Eulerian and Lagrangian approaches for predicting the behaviour of discrete particles in turbulent flows, Progress in Energy and Combustion Science, 25, pp. 133-159, 1999.

[12] Busch, B., Ferron, G., Karg, E., Silberg, A. \& Heyder, G., The growth of atmospheric particles in moist air, Journal of Aerosols Science, 26, pp. S435-S436, 1995.

[13] Einberg, G. \& Holmberg, S., Characteristics of Particles and their Behaviour in Ventilation Air, International Journal of Ventilation, 2,

[14] Zhao, B. \& Guan, P., Modelling particle dispersion in personalized ventilated room, Building and Environment, 37 , pp. 1500-1512 , 2005. 
[15] Norris, L.H. \& Reynolds, W.C., Turbulent channel flow with a moving wavy boundary, Department of Mechanical Engineering, FM-10, pp. 300-325, 1975.

[16] Bouilly, J., Etude de l'impact de la pollution particulaire sur la qualité de l'air intérieur en site urbain, Thèse présentée devant l'Université de La Rochelle (Chapter 3), pp. 115, 2003.

[17] Lai, A.C.K., Particle deposition indoors: a review, Indoor Air, 2003. 